\title{
INFLUÊNCIA DA RESOLUÇÃO DO MODELO DIGITAL DE ELEVAÇÃO NA DETERMINAÇÃO DO ÍNDICE TOPOGRÁFICO DE UMIDADE E NA CAPACIDADE DE PREDIÇÃO DOS TEORES CARBONO ORGÂNICO DO SOLO
}

\author{
INFLUENCE OF THE RESOLUTION OF DIGITAL ELEVATION MODEL IN DETERMINING THE TOPOGRAPHIC \\ WETNESS INDEX AND ABILITY TO PREDICT THE SOIL ORGANIC CARBON CONTENT
}

\author{
Viviane Capoane \\ Universidade Federal de Santa Maria (UFSM), Santa Maria, RS, Brasil, capoane@gmail.com
}

Tales Tiecher

Universidade Regional Integrada do Alto Uruguai e das Missões (URI),Erechim, RS, Brasil, tales.t@hotmail.com

Jimmy Walter Rasche Rasche Alvarez

Universidad Nacional de Asunción (UNA), Assunção, Paraguai, jwrasche@yahoo.com.ar

André Pellegrini

Universidade Tecnológica Federal do Paraná (UTFPR), Curitiba, PR, Brasil, andrepellegrini@yahoo.com.br

Gilmar Luiz Schaefer

Universidade Federal de Santa Maria (UFSM), Santa Maria, RS, Brasil, gilmar.geo08@gmail.com

Leonardo José Cordeiro Santos

Universidade Federal do Paraná (UFPR), Curitiba, PR, Brasil, santos@ufpr.br

Danilo Rheinheimer dos Santos

Universidade Federal de Santa Maria (UFSM), Santa Maria, RS, Brasil, danilonesaf@gmail.com

\begin{abstract}
RESUMO
Neste trabalho foram gerados Modelos Digitais de Elevação (MDE's) utilizando dados altimétricos de duas fontes; carta topográfica na escala 1:50.000 e, levantamento altimétrico com um aparelho de posicionamento cinemático em tempo real (RTK) na escala 1:10.000. O objetivo foi investigar a dependência da escala na determinação do índice Topográfico de Umidade (ITU) para uma bacia hidrográfica do planalto do Rio Grande do Sul e, avaliar o potencial deste índice em discretizar os teores de Carbono Orgânico Total (COT) do solo. O efeito das diferentes resoluções sobre a distribuição espacial do ITU foi avaliada por meio de estatística descritiva. A análise entre os teores de carbono e estoque de carbono nos 57 pontos de amostragem de solo com os valores do índice topográfico de umidade foi feita por correlação linear. Os resultados obtidos comprovam que a utilização de MDE's gerados com dados altimétricos de baixa resolução espacial são inadequados para discretizar às áreas com maior probabilidade de atingirem o estado de saturação hídrica, devido, principalmente, as limitações de acurácia posicional e vertical. A utilização de MDE's gerados com dados altimétricos mais refinados, RTK, discretizou satisfatoriamente às áreas propensas à saturação hídrica. Os coeficientes de correlação entre o COT e estoque de carbono com os valores do ITU gerado com dados do RTK foram estatisticamente significativos, comprovando o grande potencial deste índice na predição dos teores de carbono nas cinco camadas de solo avaliadas e do estoque de $\mathrm{C}$ até $60 \mathrm{~cm}$ para a bacia hidrográfica estudada.
\end{abstract}

Palavras-chave: Resolução; Atributos Topográficos; Áreas Úmidas, Carbono.

\footnotetext{
ABSTRACT

In this paper Digital Elevation Models (DEM's) were generated using altimetry data from two sources; topographic maps at 1:50.000 scale and altimetry surveys with Real Time Kinematic (RTK) positioning at scale 1:10.000. The objective was investigate the dependence of the scale in determining the Topographic Wetness Index (TWI) for a watershed plateau of Rio Grande do Sul, and evaluate the potential of this index discretize the levels of Total Organic Carbon (TOC) of the soil. The effect of different resolutions on the spatial distribution of TWI was evaluated using descriptive statistics. The analysis of the carbon content and carbon stock in 57 soil sampling points, with the values of the topographic wetness index content was made by linear correlation. The results show that the use of DEMs created with altimetry data from low spatial resolution are inadequate to discretize the area most likely to reach the state of water saturation, mainly due to limitations of positional and vertical accuracy. The use of DEM's created with the best altimetry data, RTK, delimited satisfactorily to areas prone to water saturation. The correlation coefficients between TOC and carbon stock with the values generated by the TWI-RTK data were statistically significant, demonstrating the great potential of this index in the prediction of the carbon in the five layers of soil evaluated and the stock of $C$ to $60 \mathrm{~cm}$ for the watershed studied.
} 
Keywords: Resolution; Topographic Attributes; Wetlands; Carbon.

Artigo recebido para publicação em outubro de 2014

Artigo aceito para publicação em agosto de 2015

\section{INTRODUÇÃO}

A bacia hidrográfica é a unidade básica de planejamento para a compatibilização da preservação dos recursos naturais e da produção agropecuária. Elas possuem características ecológicas, geomorfológicas e sociais integradoras, o que possibilita a abordagem holística e participativa envolvendo estudos interdisciplinares para o estabelecimento de formas de gestão e desenvolvimento inerentes às condições ecológicas locais e regionais (ATTANASIO et al., 2006). Para a manutenção da saúde ambiental de bacias hidrográficas agrícolas, áreas com saturação hídrica temporária ou permanente exercem importante função do ponto de vista hidrológico, ecológico e geomorfológico (ZAKIA et al., 2006). Essas áreas possuem maior probabilidade de atingirem o estado de saturação do solo além de apresentar forte relação com a dinâmica do lençol freático, devido à expansão e contração durante eventos pluviométricos.

A identificação e a distribuição espacial das áreas com saturação hídrica temporária ou permanente pode ser feita utilizando o índice topográfico de umidade, o qual é definido pela relação entre a declividade local e a área de contribuição específica de montante, possuindo, portanto variação espacial significativa. Vários índices de umidade derivados de Modelos Digitais de Elevação (MDE's) foram propostos (IVERSON et al., 1997; SUMMERELL et al., 2004; MURPHY et al., 2009), mas o mais conhecido é o índice topográfico de umidade (ITU), definido como $\ln (\mathrm{As} / \tan \beta)$, onde As é a área de contribuição específica por unidade de largura ortogonal à linha de fluxo (m2 m-1), ou seja, uma medida que representa o volume potencial do escoamento superficial em dado ponto da vertente e, $\beta$ é o declive local (BEVEN \& KIRKBY, 1979; WILSON \& GALLANT, 2000). A área de contribuição específica está relacionada com o conceito de escoamento acumulado e leva em conta a complexidade da encosta, ou seja, descreve a tendência de um local para receber a água superficial. A inclinação é um parâmetro que descreve a tendência para evacuar a água (GRUBER \& PECKHAM, 2008). Este 
índice é, portanto uma medida relativa da disponibilidade em longo prazo de umidade do solo de um determinado local na paisagem. No caso das vertentes convexas para que o fluxo acumulado seja divergente, a área de contribuição específica tende a diminuir. Para vertentes côncavas, a área de contribuição específica tende a aumentar, dando origem a um aumento rápido do fluxo acumulado à jusante, definida como área de contribuição específica.

A crescente disponibilidade de MDE's e o advento de ferramentas de análise computadorizada do terreno tornou mais fácil a quantificação dos atributos topográficos de uma paisagem. Isto facilitou o desenvolvimento de pesquisas em diversas áreas das ciências ambientais, como agricultura, hidrologia, biologia, geografia e engenharias, as quais utilizam variáveis dependentes da topografia (RUHOFF et al., 2011). Atualmente, índices topográficos derivados de MDE's em ambiente do Sistema de Informação Geográfica (SIG) têm sido amplamente utilizados (MOORE et al., 1991; GRUBER \& PECKHAM, 2008). No entanto, diferentemente de alguns países, não existe no Brasil um órgão responsável pela elaboração e normatização dos procedimentos utilizados na criação de MDE's os quais, normalmente, são produzidos pelos próprios usuários e pouca atenção é dada às suas limitações como fonte de informação espacial (CHAGAS et al., 2009). A fonte de dados mais comum para a geração de MDE's no Brasil ainda são as curvas de nível e pontos cotados e, de forma complementar, a rede hidrográfica, obtidas em cartas topográficas, principalmente aquelas elaboradas pela Diretoria do Serviço Geográfico (DSG) do Exército na década de 70. No caso do Rio Grande do Sul, cartas topográficas na escala 1:50.000 cobrem todo o Estado e podem ser facilmente adquiridas, já escalas de maior detalhe restringem-se a pequenas áreas. Por isso os dados altimétricos mais utilizados para geração de MDE's e extração de atributos topográficos são os na escala 1:50.000 e, mais recentemente TOPODATA $(30 \mathrm{~m})$ e SRTM $(90 \mathrm{~m})$. Entretanto, essa escala não é adequada para estudos mais detalhados.

Diante do exposto, o objetivo do presente trabalho foi investigar a dependência da escala e fonte de dados do modelo digital de elevação na determinação do índice topográfico de umidade para uma bacia 
hidrográfica do planalto do Rio Grande do Sul e, avaliar o potencial deste índice em discretizar os teores de carbono orgânico do solo.

\section{MATERIAL E MÉTODOS}

\section{Local do estudo}

O presente trabalho foi desenvolvido em uma bacia hidrográfica $(\mathrm{BH})$ agrícola localizada no município de Júlio de Castilhos, região central do Estado do Rio Grande do Sul. A BH possui área de captação de 80,2 ha (Figura 1). O relevo local é homogêneo, formado de modo geral por colinas suaves, bem arredondadas, esculpidas em rochas sedimentares correspondentes à Formação Tupanciretã. A classe de solo predominante é Argissolo. Os principais usos da terra são a integração lavoura-pecuária, com cultivo de soja e milho sob sistema plantio direto no verão e pastoreio do gado em áreas de aveia e azevém no inverno.

\section{Geração dos MDE's e determinação do ITU}

Os modelos digitais de elevação foram gerados com dados da carta topográfica da Diretoria do Serviço Geográfico do Exército (1976) na escala 1:50.000 e, de um levantamento altimétrico realizado com um aparelho de posicionamento cinemático em tempo real (Real Time Kinematic - RTK) no ano de 2012.

O MDE obtido a partir de dados da carta topográfica na escala de 1:50.000 foi elaborado utilizando os dados relativos às curvas de nível, pontos cotados e rede de drenagem, referente a Folha Val de Serra (SH.22.V.C.I-4), mantendo-se a equidistância vertical de $20 \mathrm{~m}$. A base de dados foi obtida no formato digital de um banco de dados da Universidade Federal do Rio Grande do Sul (HASENACK \& WEBER, 2010). O MDE obtido a partir de dados do levantamento com RTK foi gerado pela interpolação de 4.039 pontos cotados, sendo a resolução espacial escolhida 5 m, escala 1:10.000. 
A interpolação dos dados de ambas as fontes foi feita no software ArcGis 10, utilizando o método Topogrid, desenvolvido para estudos hidrológicos (HUTCHINSON, 1989). O MDE Topogrid utiliza o método de interpolação de diferenças finitas, combinando interpolação local com o método do inverso do quadrado da distância e interpolação global que utiliza uma superfície de continuidade, como o interpolador Kriging (ESRI, 2010). O procedimento é chamado Topo to Raster (ArcGis10 $\rightarrow$ ArcToolbox $\rightarrow$ Spatial Analyst Tools $\rightarrow$ Interpolation $\rightarrow$ Topo to Raster $).$

Considerando que os algoritmos de distribuição de fluxo são elementos chaves em estudos hidrológicos e que o ArcGis 10 considera o fluxo unidimensional utilizando um único algoritmo de fluxo, o Deterministic 8 (D8) (O CALLAGHAN \& MARK, 1984), foi feita a migração para o software SAGA GIS 2.0.8. para a confecção do mapa com o índice topográfico de umidade. Este software é muito mais evoluído neste aspecto, apresentando inúmeras opções de algoritmos de distribuição de fluxo (D8; FD8Q - QUINN et al., 1991; MD1 - SEIBERT \& MCGLYNN, 2007; FD8F - FREEMAN, 1991; DMF, Dœ - TARBOTON, 1997; Rho8 - FAIRFIELD \& LEYMARIE, 1991; dentre outros), pois considera o fluxo bidimensional.

\section{Amostragem de solo e análises laboratoriais}

O índice topográfico de umidade tem sido bastante utilizado para a predição da matéria orgânica do solo, pois estima um balanço entre acúmulo de água e condições de drenagem em escala local (PEI, et al., 2010, HANCOCK et al., 2010). A fim de validar o potencial do ITU na predição da matéria orgânica do solo, foram coletadas amostras em diferentes pontos da bacia hidrográfica. A coleta foi realizada nos meses de junho, julho e agosto de 2013 em 57 pontos de amostragem (Figura 1). Em cada ponto foram coletadas amostras nas profundidades 0-5, 5-10, 10-20, 20-40, 40-60 cm, perfazendo um total de 285 amostras.

Após a coleta, as amostras de solo foram colocadas em sacos plásticos, acondicionadas em caixas e transportadas até o Laboratório de Química e Fertilidade do Solo da Universidade Federal de Santa Maria, onde foram secas em estufa de circulação forçada de ar à $\quad 50^{\circ} \mathrm{C}$, peneiradas em malha de 2 mm e armazenadas em potes plásticos até o momento das análises físicas e químicas. A densidade do 
solo foi determinada pelo método do anel volumétrico, conforme Embrapa (1997) e os resultados foram utilizados para o cálculo do estoque de carbono. 0 teor de carbono orgânico total, que constitui cerca de 58\% da massa matéria orgânica do solo, de acordo com Bayer (1996), foi estimado pelo método de combustão úmida Walkley \& Black (1934), adaptado (EMBRAPA, 1997) e, os teores obtidos foram corrigidos para autoanalisador de carbono conforme metodologia proposta por Rheinheimer et al. (2008). O estoque de carbono no solo foi calculado conforme metodologia proposta por Fernandes \& Fernandes (2008), para solos sob diferentes condições de manejo, corrigindo os estoques de carbono para uma mesma massa de solo.

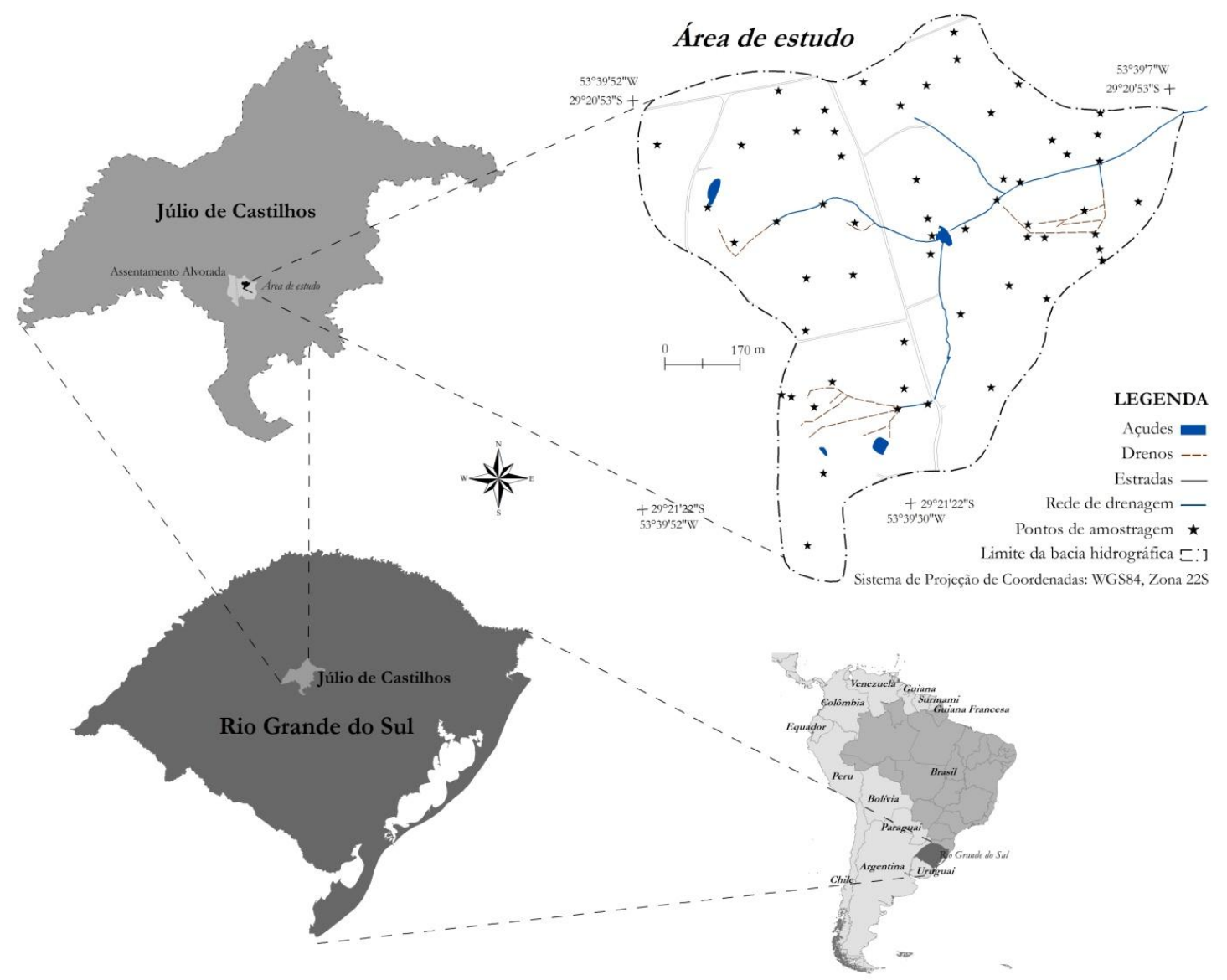

Figura 1. Mapa com a localização da área estudada e dos pontos de amostragem de solo.

\section{Mapa temático de uso da terra}

O mapa temático de uso da terra foi confeccionados no software ArcGis 10, utilizando uma imagem pancromática do satélite Quick Bird de 22/02/2008, com resolução espacial de 60 cm. Após a 
delimitação dos polígonos com as diferentes classes de uso, foi feita atualização em campo para o ano de 2013.

\section{Análise estatística}

A análise dos dados dos índices topográficos de umidade entre as diferentes fontes de dados e escala foi feita por estatística descritiva. A análise entre os teores de carbono e estoque de carbono nos 57 pontos de amostragem de solo, com os valores do índice topográfico de umidade foi feita por correlação linear.

\section{RESULTADOS E DISCUSSÃO}

Na base de dados carta topográfica o valor do ITU variou de 7 a 20, sendo que o desvio padrão foi de 1,53 (Figura 2A e Tabela 1). Na base de dados RTK o ITU variou de 4 a 20, sendo que o desvio padrão foi de 1,93 (Figura 2B e Tabela 1). Devido as limitações de acurácia posicional e vertical da carta topográfica na escala 1:50.000 as variáveis do terreno derivadas possuem baixo detalhamento, logo, muitas nuances da paisagem não são representadas. Conforme Wolock \& McCabe (2000), isso decorre da baixa resolução do MDE que provoca a diminuição no declive médio e aumento na área de contribuição. Assim, se forem utilizados dados topográficos como TOPODATA (30 m), SRTM (90 m) ou mesmo cartas topográficas na escala 1:50.000 (20 m), que tem baixa resolução espacial, a determinação do ITU não será precisa, ou seja, excederá a escala em que os processos hidrológicos e geomorfológicos ocorrem. A diferença de área de captação foi de 10,08 ha entre as bases carta topográfica (90,30 ha) e RTK (80,22 ha). O MDE com resolução espacial de 5 m obtido do levantamento altimétrico com GPS dinâmico RTK representou melhor as feições do terreno e, consequentemente as áreas com maior potencial de atingirem o estado de saturação hídrica (Figura 2B).

\begin{tabular}{ccc}
\hline Estatística descritiva & Carta topográfica & RTK \\
\hline Mínimo & 7 & 4 \\
Máximo & 20 & 20 \\
Média & 10,33 & 7,94
\end{tabular}




\section{Desvio padrão $\quad 1,53 \quad 1,96$}

Tabela 1. Estatística descritiva do índice topográfico de umidade nas duas bases de dados analisadas.

De acordo com os mapas gerados, a cor azul mais escuro representa as áreas com maior potencial de umidade do solo, localizadas principalmente em vertentes côncavas e áreas próximas a cursos d'água. As cores intermediárias representam as pendentes de curvatura convergente em que os fluxos tendem a se concentrar, aumentando a umidade do solo, essas áreas estão presentes, principalmente, no topo e terço médio e base das encostas. As áreas em azul claro indicam baixa umidade do solo.

Observando a Figura 2B e comparando com a Figura 3A, onde são apresentadas as classes de uso da terra fica evidente que o ITU gerado com um MDE de alta resolução espacial tem capacidade muito maior de discretizar as áreas com potencial de saturação hídrica ou baixa umidade do solo quando comparada ao ITU gerado com dados da carta topográfica na escala 1:50.000 (Figura 2A). Por exemplo, comparando a classe de uso da terra área úmida (banhados) localizada no fundo de vale (Figura 3A), com os locais em azul mais escuro do mapa com o ITU (Figura 2B), percebe-se a correlação entre ambos, ou seja, a predição automática do ITU coincidiu com os locais com grande concentração de umidade, solos com caráter hidromórfico da bacia hidrográfica estudada. As áreas com menor umidade (Figura 2B), representadas pela cor azul mais clara estão localizadas em vertentes divergentes, estradas e, em locais com declive mais acentuado.
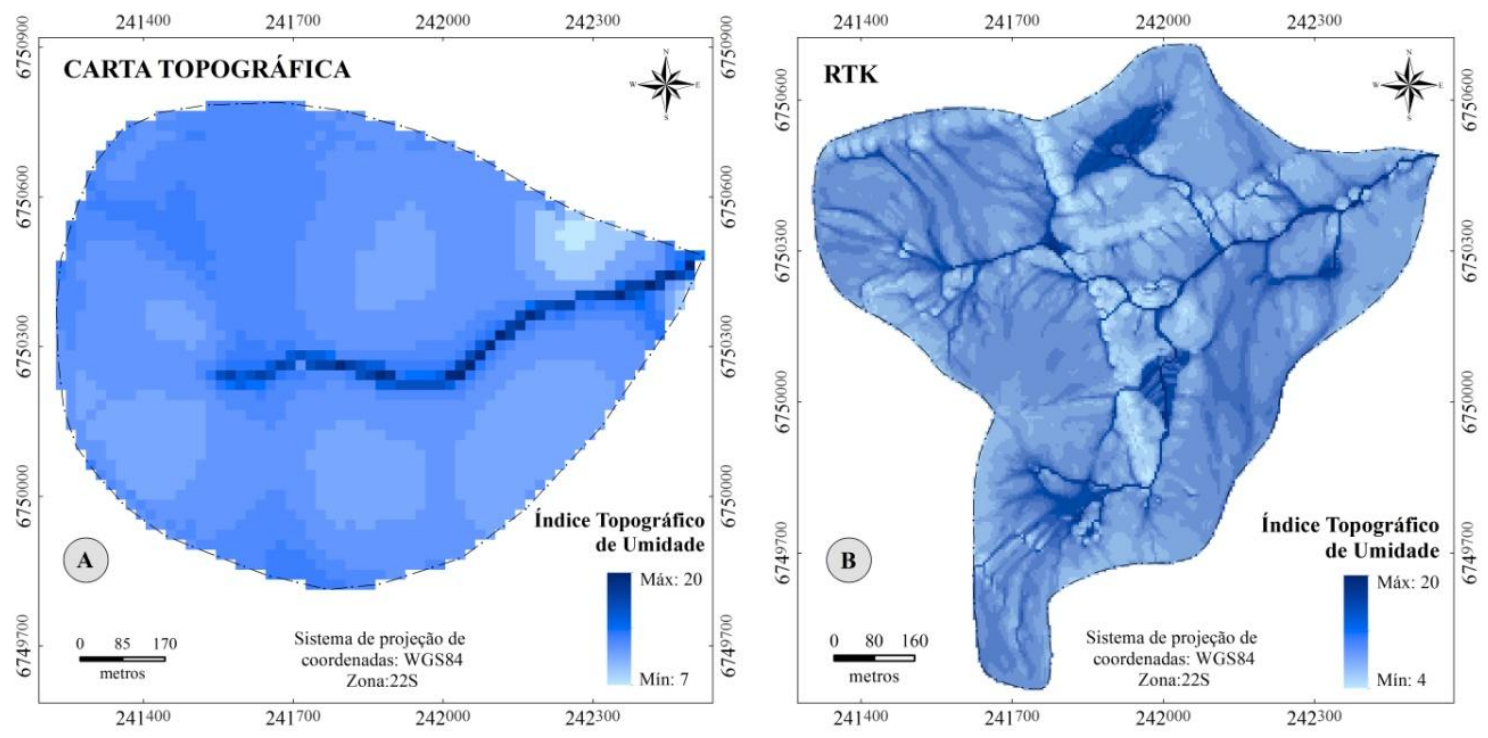
Figura 2. Distribuição espacial do índice topográfico de umidade do solo da bacia hidrográfica estudada: A) carta topográfica 1:50.000; B) levantamento planialtimétrico com RTK - Real Time Kinematic.
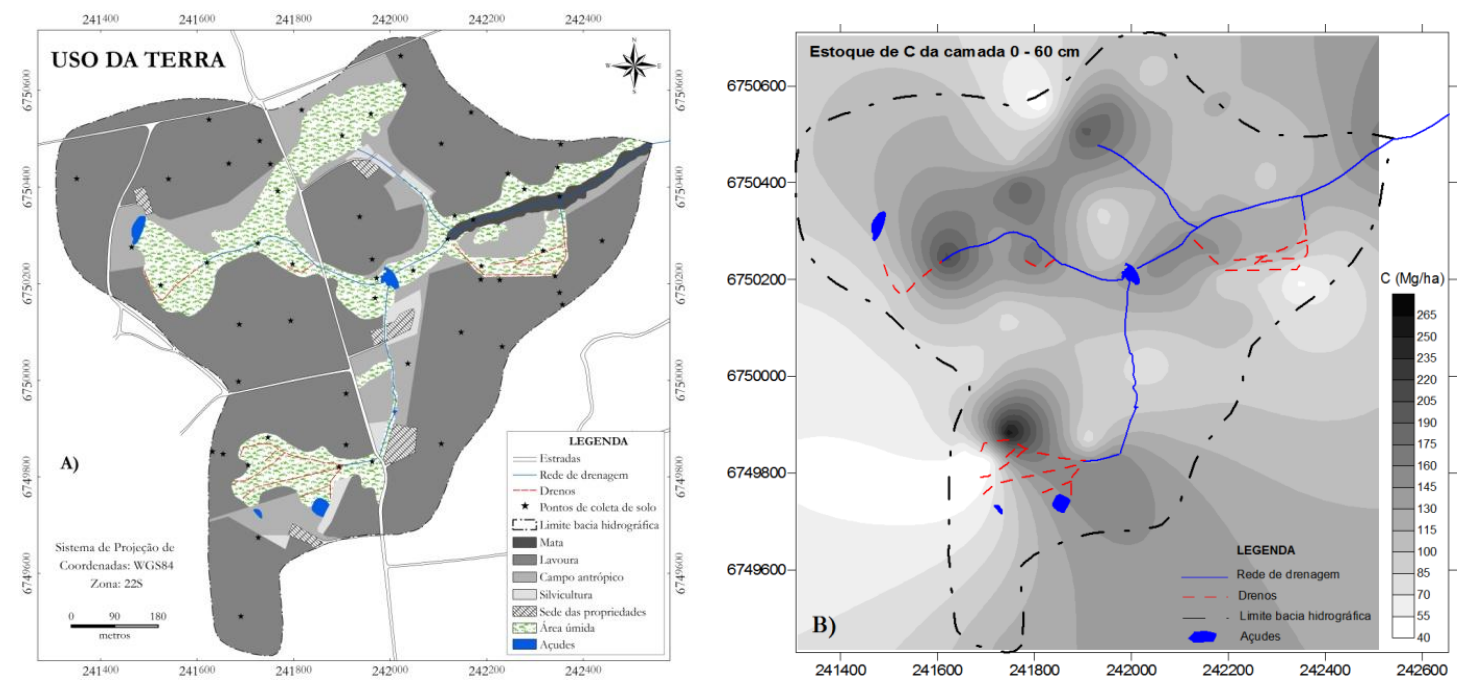

Figura 3. A) Mapa com as classes de uso da terra da bacia hidrográfica estudada; B) Estoque de carbono do solo na camada $0-60 \mathrm{~cm}$.

Os coeficientes de correlação entre COT e estoque de C com o atributo do terreno ITU foram altos, com valores estatisticamente significativos (Tabela 2). Os resultados obtidos comprovam a forte influência do relevo na redistribuição de carbono na paisagem bem como a eficácia do índice topográfico de umidade como preditor do teor de carbono nas 5 camadas de solo avaliadas e do estoque de C até $60 \mathrm{~cm}$ (Figura 3B) para a bacia hidrográfica estudada.

\begin{tabular}{|c|c|c|}
\hline \multirow{2}{*}{ Parâmetro } & Profundidade & ITU \\
\hline & $\mathrm{em}$ & $\mathbf{r}$ \\
\hline \multirow{5}{*}{$\mathrm{COT} \mathrm{g} \mathrm{kg}^{-1}$} & $0-5$ & $* 0,704$ \\
\hline & $5-10$ & $* 0,696$ \\
\hline & $10-20$ & $* 0,516$ \\
\hline & $20-40$ & $* 0,590$ \\
\hline & $40-60$ & $* * 0,310$ \\
\hline Estoque $\mathrm{C} \mathrm{Mg} \mathrm{ha}{ }^{-1}$ & $0-60$ & $* 0,659$ \\
\hline
\end{tabular}

Tabela 2. Correlação entre o índice topográfico de umidade com os teores de carbono orgânico total nas cinco profundidades e estoque de carbono da camada $0-60 \mathrm{~cm} .{ }^{*}$ significativo a $\mathrm{p}<0.001$; significativo a $\mathrm{p}<0.05$

Não foi efetuada a análise de correlação linear entre os teores de COT e estoque de C com os valores do ITU gerado com dados da carta topográfica, pois este não apresentou consistência geomorfológica 
ou hidrológica, ou seja, a imagem matricial não representou fidedignamente os divisores de água da bacia hidrográfica estudada nem os talvegues e elementos côncavos e convexos. Além disso, alguns pontos de amostragem de solo ficaram fora do limite da bacia hidrográfica, como pode ser visualizado na Figura 4.
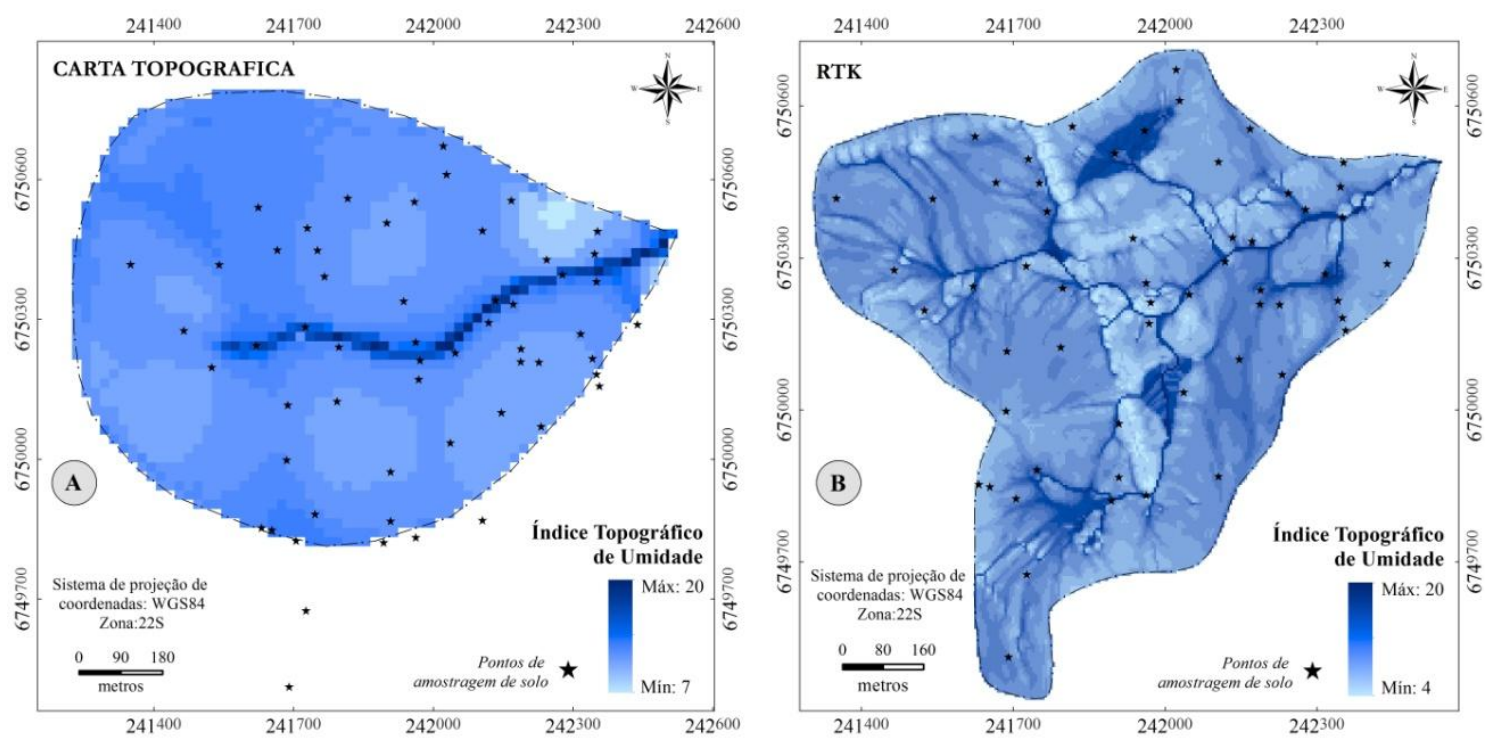

Figura 3. Mapa do índice topográfico de umidade e pontos de amostragem de solo nas diferentes bases de dados.

\section{CONSIDERAÇÕES FINAIS}

Foi comprovado neste trabalho que a utilização de um modelo digital de elevação gerado com dados altimétricos da carta topográfica na escala 1:50.000 é inadequado para discretizar as áreas com maior probabilidade de atingirem o estado de saturação hídrica do solo, devido, principalmente, as limitações de acurácia posicional e vertical. A utilização de um modelo digital de elevação gerado com dados altimétricos mais refinados discretizou satisfatoriamente às áreas propensas à saturação hídrica.

Os coeficientes de correlação entre o COT e estoque de $\mathrm{C}$ com os valores do ITU foram estatisticamente significativos, comprovando o grande potencial deste índice na predição dos teores de carbono nas cinco camadas de solo avaliadas e, do estoque de $\mathrm{C}$ até $60 \mathrm{~cm}$, para a bacia hidrográfica estudada. 


\section{REFERÊNCIAS}

ATTANASIO, C. M.; LIMA, W. P.; GANDOLFI, S.; ZAKIA, M. J. B.; VENEZIANI-JÚNIOR, J. C. T. Método para a identificação da zona ripária: microbacia hidrográfica do Ribeirão São João (Mineiros do Tietê, SP). Scientia Forestalis, n.71, p. $131-140.2006$.

BAYER, Cimélio. Dinâmica da matéria orgânica em sistemas de manejo do solo. 240 f. Tese (Doutorado em Ciência do Solo) - Departamento de Solos, Universidade Federal de Rio Grande do Sul, Porto Alegre, 1996.

BEVEN, K. J.; KIRKBY, M. J. A physically based, variable contributing area model of basin hydrology. Hydrologic Science Bulletin, v.24, n.1, p.43-69. 1979.

CHAGAS, C. S.; FERNANDES FILHO, E. I.; ROCHA, M. F.; CARVALHO JÚNIOR, W.; SOUZA NETO, N. C. Avaliação de modelos digitais de elevação para aplicação em um mapeamento digital de solos. Revista Brasileira de Engenharia Agrícola e Ambiental, v.14, n.2, p.218-226. 2010.

EMBRAPA - Empresa Brasileira de Pesquisa Agropecuária. Centro Nacional de Pesquisa de Solos. Manual de métodos de análise de solos. 2 ed. Rio de Janeiro, Embrapa Solos, p. 212, 1997.

ESRI, 2011. ArcGIS Desktop: Release 10. Redlands, CA: Environmental Systems Research Institute.

FAIRFIELD, J.; LEYMARIE, P. Drainage networks from grid digital elevation models. Water Resources Research, v.27, p.709-717. 1991 .

FERNANDES, F. A.; FERNANDES, A. H. B. M. Calculo dos estoques de carbono do solo sob diferentes condições de manejo. Corumbá: Embrapa Pantanal, dez. 2008. (Embrapa Pantanal. Comunicado Técnico, 69). Disponível em: <http://www.cpap.embrapa.br/publicacoes/download.php?arq_pdf=COT69>. Acesso em: 27 fev. 2013.

FREEMAN, G. T. Calculating catchment area with divergent flow based on a regular grid. Computers and Geosciences, v.17, p.413-422. 1991 .

GRUBER, S.; PECKHAM, S. Land-surface parameters and objects in hydrology. In: HENGL, T.; REUTER, H. I. (eds.) Geomorphometry: concepts, software, applications. Amsterdam: Elsevier, 2008, p.171-194.

HANCOCK, G. R.; MURPHY, D.; EVANS, K. G. Hillslope and catchment scale soil organic carbon concentration: An assessment of the role of geomorphology and soil erosion in an undisturbed environment. Geoderma, v.155, n.36-45. 2010.

HASENACK, H.; WEBER, E. (org.). Base cartográfica vetorial contínua do Rio Grande do Sul - escala 1:50.000. Porto Alegre, UFRGS-IB-Centro de Ecologia, 2010. 1 DVD-ROM (Série Geoprocessamento, 3).

HUTCHINSON, M. F. A new procedure for gridding elevation and stream line data with automatic removal of spurious pits. Journal of Hydrology, v.106, n.3-4, p.211-232. 1989.

IVERSON, L. R. et al. A GIS-derived integrated moisture index to predict forest composition and productivity of Ohio forests (U.S.A.). Landscape Ecology, v.12, p.331-348. 1997.

MOORE, I. D.; GRAYSON, R. B.; LADSON, A. R. Digital terrain modelling: a review of hydrological, geomorphological, and biological applications. Hydrologic Processes, v.5, n.1, p.3-30. 1991. 
MURPHY, P. N. C.; OGILVIE, J.; ARP, P. Topographic modelling of soil moisture conditions: a comparison and verification of two models. European Journal of Soil Science, v.60, p.94-109. 2009.

O'CALLAGHAN, J. F.; MARK, D. M. The extraction of drainage networks from digital elevation data. Computer Vision, Graphics and Image Processing, v.28, n.3, p.328-344. 1984.

PEI, T.; QIN, C-Z.; ZHU, A-X; YANG, L.; LUO, M.; LI, B.; ZHOU, C. Mapping soil organic matter using the topographic wetness index: A comparative study based on different ow-direction algorithms and kriging methods. Ecological Indicators, v.10, p.610-619. 2010.

QUINN, P.; BEVEN, K.; CHEVAlLIER, P.; PLANCHON, O. The prediction of hillslope flow paths for distributed hydrological modeling using digital terrain models. Hydrological Processes, v.5, p.59-80. 1991.

RHEINHEIMER, D. S.; CAMPOS, B. C.; GIACOMINI, S. J.; CONCEIÇÃO, P. C.; BORTOLUZZI, E. C. Comparação de métodos de determinação de carbono orgânico total no solo. Revista Brasileira de Ciência do Solo, v. 32, p. 435-440. 2008.

RUHOFF, A. L.; CASTRO, N. M. R.; RISSO, A. Numerical modelling of the topographic wetness index: an analysis at different scales. International Journal of Geosciences, v.2, n.4, p. 476-483. 2011.

SAGA - System for Automated Geoscientific Analyses, Version: 2.0.2. 2005. http://www.saga-gis.org

SUMMERELL, G. K. Dowling, T. I.; Wild, J. A.; Beale, G. FLAG UPNESS and its application for mapping seasonally wet to waterlogged soils. Australian Journal of Soil Research, v.42, n.2, p.155-162. 2004.

TARBOTON, D. G. A new method for the determination of flow directions and upslope areas in grid digital elevation models. Water Resources Research, v.33, p.309-319, 1997.

ZAKIA, M. J. B.; FERRAZ, F. F. B.; RIGHETTO, A. M.; LIMA, W. P. Delimitação da Zona Ripária em uma microbacia. In: LIMA, W.P.; ZAKIA, M.J.B. (Org.). As florestas plantadas e a água. São Carlos: RiMa, 2006. p.89-106.

WALKLEY, A.; BLACK, I. A. An examination of the Degtjareff method for determining soil organic matter, and a proposed modification of the chromic acid titration method. Soil Science, v. 37, p. 29-38. 1934.

WILSON, J. P.; GALLANT, J. C. Terrain Analysis: Principles and Applications. John Wiley \& Sons Inc., New York, 2000 .

WOLOCK, D. M.; MCCABE, G. J. Differences in topographic characteristics computed from 100 and 1000 m resolution digital elevation models. Hydrological Processes, v.14, p.987-1002. 2000. 